\title{
AN INEQUALITY FOR SOME NONNORMAL OPERATORS
} TAKAYUKI FURUTA

\author{
(Communicated by Daniel W. Stroock) \\ Dedicated to Professor Tsuneo Kanno on his sixtieth birthday \\ with respect and affection
}

ABSTRACT. An inequality of use in testing convergence of eigenvector calculations is improved. If $e_{\lambda}$ is a unit eigenvector corresponding to an eigenvalue $\lambda$ of a dominant operator $A$ on a Hilbert space $H$, then

$$
\left|\left(g, e_{\lambda}\right)\right|^{2} \leq \frac{\|g\|^{2}\|A g\|^{2}-|(g, A g)|^{2}}{\|(A-\lambda I) g\|^{2}}
$$

for all $g$ in $H$ for which $A g \neq \lambda g$. The equality holds if and only if the component of $g$ orthogonal to $e_{\lambda}$ is also an eigenvector of $A$. This result is an improvement of Bernstein's result for selfadjoint operators.

1. Statement of the results. An operator $A$ means a bounded linear operator on a complex Hilbert space $H$. An operator $A$ is called dominant if there is a real number $M_{\lambda} \geq 1$ such that

$$
\left\|(A-\lambda)^{*} x\right\| \leq M_{\lambda}\|(A-\lambda) x\|
$$

for all $x$ in $H$ and for all complex numbers $\lambda$. If there is a constant $M$ such that $M_{\lambda} \leq M$ for all $\lambda, A$ is called $M$-hyponormal. The inclusion relation of these classes of nonnormal operators is as follows:

\section{Selfadjoint $\subset$ Normal $\subset$ Quasinormal $\subset$ Subnormal $\subset$ Hyponormal $\subset M$-hyponormal $\subset$ Dominant,}

and it is well known that the inclusions above are all proper.

THEOREM 1. If $e_{\lambda}$ is a unit eigenvector corresponding to an eigenvalue $\lambda$ of a dominant operator $A$ on a Hilbert space $H$, then

$$
\left|\left(g, e_{\lambda}\right)\right|^{2} \leq \frac{\|g\|^{2}\|A g\|^{2}-|(g, A g)|^{2}}{\|(A-\lambda) g\|^{2}}
$$

for all $g$ in $H$ for which $A g \neq \lambda g$. The equality holds if and only if the component of $g$ orthogonal to $e_{\lambda}$ is also an eigenvector of $A$. The bound of the right-hand side is $\|(A-\tau) g\|^{2} /|\lambda-\tau|^{2}$ for any complex $\tau$.

The corresponding result for selfadjoint operators in Theorem 1 is shown by Bernstein [1]. In this note, we extend the Bernstein result to the class of dominant operators, wider than the one of selfadjoint operators, by appropriate modification of Bernstein [1] together with the following lemma.

Received by the editors September 9, 1987.

1980 Mathematics Subject Classification (1985 Revision). Primary 65F15; Secondary 65J10, 47B20.

Key words and phrases. Eigenvector, dominant operator. 
LEMMA 1. Let $H$ be a Hilbert space, and $A$ any operator on $H$. Then

$$
\|x\|^{2}\|A x\|^{2}-|(x, A x)|^{2}=\|x\|^{2}\|(A-\tau) x\|^{2}-|(x,(A-\tau) x)|^{2}
$$

for any vector $x$ in $H$ and any complex $\tau$.

$$
\frac{\|x\|^{2}\|A x\|^{2}-|(x, A x)|^{2}}{\|(A-\lambda) x\|^{2}} \leq \frac{\|(A-\tau) x\|^{2}}{|\lambda-\tau|^{2}}
$$

for all $x$ in $H$ for which $A x \neq \lambda x$ and all complex $\tau$ and $\lambda$.

PROOF OF LEMMA 1. (i) is already shown in [2] by straightforward calculation and (ii) is also easily obtained by using (i).

LEMMA 2. Let $H$ be a Hilbert space, $A$ a dominant operator on $H, e_{\lambda}$ a unit eigenvector of $A$ with corresponding eigenvalue $\lambda$, and let $f$ be orthogonal to $e_{\lambda}$. Then for any $g=\alpha e_{\lambda}+f$ with $\alpha$ complex, either $A g=\lambda g$ or

$$
|\alpha|^{2} \leq \frac{\|g\|^{2}\|A g\|^{2}-|(g, A g)|^{2}}{\|(A-\lambda) g\|^{2}} .
$$

ProOF OF LEMMA 2. First of all, we have $(A-\lambda) g=(A-\lambda) f$ and

$$
\begin{aligned}
(g,(A-\lambda) g) & =(g,(A-\lambda) f)=\left(\alpha e_{\lambda},(A-\lambda) f\right)+(f,(A-\lambda) f) \\
& =\alpha\left((A-\lambda)^{*} e_{\lambda}, f\right)+(f,(A-\lambda) f) \\
& =(f,(A-\lambda) f)
\end{aligned}
$$

because the hypothesis $(A-\lambda) e_{\lambda}=0$ yields $(A-\lambda)^{*} e_{\lambda}=0$ by the definition of dominant operator $A$. Put $B=A-\lambda$. Since $B g=B f$ and $(g, B g)=(f, B f)$, by Lemma 1 and the Schwarz inequality, we have

$$
\begin{aligned}
\frac{\|g\|^{2}\|A g\|^{2}-|(g, A g)|^{2}}{\|(A-\lambda) g\|^{2}} & =\frac{\|g\|^{2}\|(A-\lambda) g\|^{2}-|(g,(A-\lambda) g)|^{2}}{\|(A-\lambda) g\|^{2}} \\
& =\frac{\left(|\alpha|^{2}+\|f\|^{2}\right)\|B f\|^{2}-|(f, B f)|^{2}}{\|B f\|^{2}} \\
& =|\alpha|^{2}+\frac{\|f\|^{2}\|B f\|^{2}-|(f, B f)|^{2}}{\|B f\|^{2}} \\
& \geq|\alpha|^{2} .
\end{aligned}
$$

ProOF OF THEOREM 1. Inequality is shown by Lemma 1 and Lemma 2, and it is also seen that the equality holds if and only if $f$ is an eigenvector of $B$, equivalently, $f$ is an eigenvector of $A$ in the proof of Lemma 2.

\section{REFERENCES}

1. H. J. Bernstein, An inequality for selfadjoint operators on a Hilbert space, Proc. Amer. Math. Soc. 100 (1987), 319-321.

2. G. Björck and V. Thomee, A property of bounded normal operators in Hilbert space, Ark. Mat. 4 (1963), 551-555.

Department of Mathematics, Faculty of SCIEnce, Hirosaki University, BUNKYO-CHO 3, HIROSAKI, 036 AOMORI, JAPAN 